\title{
Uso da Técnica do Micro-Hematócrito para Predição do Peso Corporal e de Caracterís- ticas Reprodutivas em Frangos de Corte ${ }^{1}$
}

\author{
Millor Fernandes do Rosário ${ }^{2}$, Vicente José Maria Savino ${ }^{3}$, Antonio Augusto Domingos Coelho ${ }^{3}$, \\ Marco Aurélio Neves da Silva ${ }^{4}$, Edmilson Martins ${ }^{5}$
}

RESUMO-O hematócrito é uma metodologia indireta utilizada no monitoramento da síndrome ascítica que poderia ser utilizada também para avaliar o peso corporal e características reprodutivas em frangos de corte. Objetivou-se com este trabalho avaliar a técnica do microhematócrito em relação ao peso corporal e características reprodutivas (fertilidade, eclodibilidade e nascimento) visando sua utilização pelos programas de melhoramento genético. Dois grupos contrastantes foram constituídos, baseados na seleção fenotípica de 630 reprodutores de 18 genótipos para a característica hematócrito. Progênies das aves selecionadas, 74 pintos não sexados (40 do grupo de hematócrito alto e 34 do grupo baixo) foram criados com manejo convencional. Os pesos corporais e os valores de hematócrito foram mensurados aos 14 e 42 dias de idade. Para avaliar as características reprodutivas, ovos de 42 matrizes ( 22 do grupo alto e 20 do baixo) foram incubados. As médias foram estimadas utilizando-se um modelo linear com o efeito fixo do grupo genético. Os pintos do grupo baixo apresentaram as maiores médias de peso corporal (305,59 e 1387,06 g), diferindo dos pintos do grupo alto os quais apresentaram médias de peso de 243,56 e 1128,11 g aos 14 e 42 dias de idade, respectivamente. Também para o valor do hematócrito os grupos diferiram significativamente. Para as características reprodutivas, não se verificou diferenças significativas entre os grupos, sendo as médias gerais para fertilidade de 93,8 e $94,9 \%$, para eclodibilidade de 98,2 e $93,9 \%$ e para nascimento de 89,3 e $82,4 \%$, respectivamente, para alto e baixo hematócrito. Concluiu-se que a técnica do micro-hematócrito pode ser empregada para avaliação do peso corporal, mas não para características reprodutivas.

Palavras-chave: ascite, eritrócito, genótipo, melhoramento animal

\section{Utilization of Micro-Hematocrit Technique on Body Weight and Reproductive Traits Prediction in Broiler Chickens}

\begin{abstract}
Hematocrit is an indirect method used to monitor ascites, which might be used also on body weight and reproductive traits evalution in broiler chickens. The aim of this paper was to evaluate the micro-hematocrit technique on body weight and reproductive traits (fertility, hatchability of fertile eggs and overall hatchability) possibiliting its aplication by poultry genetic breeding programs. Two contrasting groups were established based on phenotypic selection from 630 males and females of 18 genotypes to hematocrit characteristic. This work used 74 chicks produced by selected males and females ( 40 of the high and 34 of the low hematocrit group), which were raised under conventional conditions. Their body weights and hematocrit values were measured at 14 and 42 days of age. Eggs from 42 females ( 22 of the high and 20 of the low hematocrit group) were hatched to evaluate the reproductive traits. Means were estimated by a linear model with genetic group as fixed effect. Broilers of the low group showed highest values of body weight at 14 and 42 days of age (305.59 and $1,387 \mathrm{~g}$, respectively), differing from those of the high group, which weighted 243.56 and $1,128 \mathrm{~g}$. The groups differed also significantly for hematocrit value. There were no significant differences between the two groups for reproductive traits, and the general averages were 93.8 and $94.9 \%$ for fertility, 98.2 and $93.9 \%$ for hatchability of fertile eggs and 89.3 and $82.4 \%$, for overall hatchability respectively, for high and low groups. In conclusion, the hematocrit technique may be used to evaluate body weight, but not to evaluate reproductive traits.
\end{abstract}

Key Words: animal breeding, ascites, erythrocyte, genotype

\section{Introdução}

Em 1920, os frangos de corte levavam 120 dias para atingir $1500 \mathrm{~g}$ depeso corporal, porém, em 1980 esse tempo reduziu-se para 44 dias e mais recentemente, em 1998, apenas 33 dias para atingir o mesmo peso (Albers, 1998).
A execução de programas de melhoramento genético que buscaram máxima velocidade de ganho de peso, alta eficiência alimentar, maior rendimento de carcaça, menor deposição de gordura, além de melhor produtividade das matrizes (número de pintos produzidos por matriz alojada) desencadearam algu-

\footnotetext{
1 Trabalho apresentado no $8^{\circ}$ Simpósio Internacional de Iniciação Científica da Universidade de São Paulo, sob a forma de resumo. ${ }^{2}$ Bolsista PIBIC/CNPq - Escola Superior de Agricultura Luiz de Queiroz - Departamento de Genética - Av: Pádua Dias, 11 - Caixa Postal 83 - CEP: 13400-970 - Piracicaba/SP. E.mail: mfrosari@esalq.usp.br

${ }^{3}$ Docente - Departamento de Genética - ESALQ/USP. E.mail: poultry@esalq.usp.br

${ }^{4}$ Bolsista CAPES (MS) - Departamento de Genética - ESALQ/USP.

5 Técnico de laboratório - Departamento de Genética - ESALQ/USP.
} 
mas síndromes fisiológicas, dentre as quais, destacam-se o estresse calórico, a morte súbita e a ascite. (Sanchez et al., 2000). O mecanismo e fatores envolvidos no desenvolvimento da síndrome ascítica são descritos por Gonzales \& Macari (2000).

O valor hematócrito, o qual mensura a porcentagem de células sólidas sangüíneas, está diretamente ligado ao número de elementos figurados sangüíneos, dentre eles citam-se hemácias, basófilos, eosinófilos, heterófilos, leucócitos, monócitos, plaquetas. Segundo Sturkie \& Griminger (1986) os valores de hematócrito estão entre 29 a $48 \%$ e 25,5 a $31 \%$ para machos e fêmeas, respectivamente.

O número de hemácias pode ser influenciado pela idade, sexo, hormônios, hipóxia e outros fatores, sendo que, em galinhas, seu valor está entre 3,8 a 3,26 e entre 2,72 a 3,02 milhões de hemácias por milímetro cúbico para machos e fêmeas, respectivamente (Sturkie \& Griminger, 1986).

As alterações no valor hematócrito podem ocorrer ou ser induzidas, sem influência no número desses elementos, devido ao aumento ou diminuição da concentração de água no plasma (hemodiluição ou hemoconcentração). O emprego de epinefrina ou estado hipotérmico tendem a hemoconcentração e o modo como é feita a amostragem do sangue, para determinação do valor hematócrito, pode levar a hemodiluição, devido a utilização de anti-coagulantes em quantidades excessivas (Sturkie \& Griminger, 1986).

O estado de saúde também pode exercer influência no valor hematócrito. Assim, Washburn \& Smyth (1968), verificaram uma redução de $15 \%$ no valor hematócrito e de $23 \%$ no número de hemácias devido a anemia normocrômica macrocítica em frangos.

Gilbert (1963) citado por Sturkie \& Griminger (1986) administrou estrógeno em galinhas adultas e verificou diminuição do volume e presumivelmente do número das hemácias e empregando tiroxina, este efeito foi prevenido. Nirmalan \& Robinson (1972) administrando estrógeno em codornas sexualmente imaturas, verificaram haver diminuição do número de hemácias de 3,2 para 1,6 e de 3,19 para 1,44 milhões de hemácias por milímetro cúbico para machos e fêmeas, respectivamente, com efeito similar para o valor hematócrito; já os andrógenos diminuíram o número de hemácias nos machos e nas fêmeas, sugerindo efeito na eritropoiese. Ao nível do mar, o emprego prolongado de andrógeno diminuiu em aproximadamente $45 \%$ o valor hematócrito de frangos (Burton \& Smith, 1972).

R. Bras. Zootec., v.31, n.3, p.1396-1402, 2002 (suplemento)
O peso corporal e a eficiência reprodutiva estão ligados, entre outros fatores, ao metabolismo oxidativo, e para que este seja eficiente há a necessidade de adequada anatomia e fisiologia do sistema cardiorespiratório, o que pode ser avaliado pela técnica do micro-hematócrito, o qual é constituído basicamente pelas hemácias, responsáveis pelo transporte de oxigênio por todo organismo da ave.

A técnica do micro-hematócrito que vem sendo empregada para monitorar a síndrome ascítica em frangos de corte (Maxwell et al., 1992; Shlosberg et al., 1992; Silversides et al., 1997), poderia também ser empregada na avaliação do peso corporal e características reprodutivas em programas de melhoramento genético, por ser de fácil execução, rápida e de baixo custo. A partir de uma amostra de sangue seria possível acompanhar o desenvolvimento corporal e, na maturidade sexual, avaliar as características reprodutivas das aves.

A fim de verificar a viabilidade da utilização e difusão da técnica do micro-hematócrito, não apenas no monitoramento da síndrome ascítica, mas em outras características de interesse econômico dos programas de melhoramento genético de aves, objetivou-se com este trabalho avaliar esta técnica para predição do peso corporal e de características reprodutivas em frangos de corte.

\section{Material e Métodos}

O presente trabalho foi realizado nas instalações do Setor de Aves do Departamento de Genética ESALQ/USP, entre os meses de junho de 1998 e janeiro de 1999. A temperatura ambiente média foi de $24,2^{\circ} \mathrm{C}$ com umidade relativa média de $60 \%$ durante o período experimental.

Utilizaram-se progênies de pintos de corte de um dia de grupos genéticos experimentais, provenientes de reprodutores selecionados para alto e baixo hematócrito (alto - GAH e baixo - GBH). A constituição dos dois grupos baseou-se na seleção fenotípica individual dos valores de hematócrito de 630 aves de 18 genótipos diferentes com 35 semanas de idades, como descrito por Rosário et al. (2000a).

Os pintos foram identificados individualmente e vacinados contra a doença de Marek ao nascer. Estes foram alojados em galpão sobre piso de concreto forrado com casca de arroz, recebendo alimento e água ad libitum e manejo convencional de criação intensiva. No sétimo dia de idade, as aves receberam 
vacinação contra as doenças de Newcastle e Gumboro na água de bebida.

Durante a criação foram utilizados três tipos de ração, contendo os seguintes níveis de proteína bruta (\%) e energia metabolizável (kcal $/ \mathrm{kg}): \mathrm{R} 1=22 \mathrm{e}$ 3050 , fornecida de 0 aos 28 dias; $\mathrm{R} 2=20$ e 3100 , fornecida dos 29 aos 42 dias e R3 = 18 e 3150 , fornecida dos 43 aos 49 dias de idade. A formulação dessas rações foi elaborada com base nas tabelas de exigências nutricionais do NRC (1994).

O peso corporal das aves e o valor hematócrito (GAH - 19 machos e 21 fêmeas e GBH - 15 machos e 19 fêmeas) foram avaliados aos 14 e 42 dias de idade. O peso corporal foi obtido por meio de pesagem individual em balança do tipo dinamômetro, com capacidade até $5 \mathrm{~kg}$ e precisão de $20 \mathrm{~g}$. O valor de hematócrito foi obtido através da técnica do microhematócrito como descrito em Rosário et al. (2000a).

Após completarem 49 dias de idade, 20 machos e 40 fêmeas foram realojados em gaiolas individuais, nas quais procedeu-se a recria até a idade de 133 dias. Nessa fase, as aves foram submetidas a restrição alimentar recebendo diariamente ração com $16 \%$ de proteína bruta e $2800 \mathrm{kcal} / \mathrm{kg}$.

A partir dos 134 dias de idade, deu-se início ao programa de estímulo à reprodução com adoção de quantidade crescente de iluminação artificial até atingir um fotoperíodo de 17 horas de luz ao dia. A ração fornecida nesta fase continha $17 \%$ de proteína bruta e $2900 \mathrm{kcal} / \mathrm{kg}$, com 3,30\% de cálcio e também foi fornecida em quantidade semanalmente crescente até o limite de 120 e 160 gramas por ave ao dia, respectivamente para machos e fêmeas. Com 161 dias de idade, iniciou-se a reprodução das aves por meio de inseminação artificial, com mistura de sêmen dos machos selecionados dentro de cada grupo.

Os ovos produzidos por 42 fêmeas ( 22 do GAH e 20 do GBH) foram coletados diariamente e estocados em câmara fria $\left(14^{\circ} \mathrm{C}\right)$ por até 7 dias. Realizaramse duas incubações sucessivas com intervalo de uma semana: a primeira aos 180 e a segunda aos 187 dias de idade das aves, num total de 157 ovos ( 85 do GAH e 72 do GBH) e 154 ovos ( 82 do GAH e 72 do GBH) na primeira e segunda incubação, respectivamente.

Aos 21 dias de incubação ocorreu o nascimento dos pintos e após foram obtidos os dados para cálculo dos parâmetros reprodutivos. O número de ovos férteis foi obtido através da abertura dos ovos do resíduo da incubação, com a observação de embriões não nascidos somado ao número de pintos nascidos.
As características reprodutivas avaliadas foram: (FERT) fertilidade $\%=(\mathrm{NOF} / \mathrm{NOI}) \times 100,($ ECLOD $)$ eclodibilidade $\%=(\mathrm{NTPS} / \mathrm{NOF})$ e (NASC) nascimento $\%=(\mathrm{NTPS} / \mathrm{NOI}) \times 100$, sendo NOF o número de ovos férteis, NOI o número de ovos incubados e NTPS o número de pintos nascidos.

O delineamento experimental foi o inteiramente casualizado, sendo a ave a unidade experimental para todas as variáveis.

$\mathrm{O}$ modelo linear utilizado para peso corporal e hematócrito foi Yikj $=\mathrm{m}+\mathrm{G} i+\mathrm{S}$ k + GS ik + e ikj, em que: $Y i k j=$ característica avaliada no i-ésimo nível de $\mathrm{G}$, no k-ésimo nível de $\mathrm{S}$ e na j-ésima repetição; $\mathrm{m}=$ média geral; $\mathrm{Gi}=$ efeito do grupo $\mathrm{i}$; $\mathrm{Sk}=$ efeito do sexo k; GS ik = efeito da interação do i-ésimo nível de G com o k-ésimo nível de $\mathrm{S}$; e ikj = erro aleatório associado a cada observação Yikj.

Os dados foram analisados pelo procedimento GLM (General Linear Models) do SAS ${ }^{\circledR}$ (1999), obtendo-se a análise de variância e quando necessário procedeu-se à comparação de médias pelo teste de Tukey $(\mathrm{P}<0,05)$.

Para as características reprodutivas utilizou-se o modelo matemático Yikj $=\mathrm{m}+\mathrm{G}$ i I k + GI ik + e ikj, em que: $Y i k j=$ característica reprodutiva no i-ésimo nível de G, no k-ésimo nível de I e na j-ésima repetição; $\mathrm{m}=$ média geral; $\mathrm{Gi}=$ efeito do grupo $\mathrm{i}$; $\mathrm{Ik}=$ efeito da incubação $\mathrm{k}$; GI ik = efeito da interação do i-ésimo nível de G com o k-ésimo nível de I; e ikj = erro aleatório associado a cada observação Yikj.

Os dados foram analisados pelo procedimento CATMOD (Modelo de Dados Categorizados) do $\mathrm{SAS}^{\circledR}$ (1999), minimizando a não normalidade do conjunto de dados, obtendo-se a análise de variância e quando necessário procedeu-se a comparação de médias pelo teste do qui-quadrado $(\mathrm{P}<0,05)$.

\section{Resultados e Discussão}

As médias para peso corporal aos 14 e 42 dias de idade foram significativamente superiores para aves GBH em relação as GAH (Tabela 1).

As médias gerais para peso corporal de $274,57 \mathrm{e}$ $1257,61 \mathrm{~g}$ aos $14 \mathrm{e} 42$ dias de idade, respectivamente, mostraram-se inferiores aos padrões de híbridos comerciais. Essas médias justificam-se pelo fato dos grupos utilizados neste trabalho apresentarem grande diversidade genética, tendo sido constituídos por aves de diferentes origens (Rosário et al., 2000a).

As médias para peso corporal aos 14 dias obtidas 
Tabela 1 - Médias e desvios-padrão de peso corporal (PC) e valor hematócrito (HCT) nos grupos de alto (GAH) e baixo $(\mathrm{GBH})$ hematócrito nas duas idades

Table 1 - Means and standard deviations of body weight (BW) and of hematocrit value (HCT) in high group (HHG) and low group (LHG) hematocrit in two different ages

\begin{tabular}{|c|c|c|c|c|}
\hline \multirow[t]{4}{*}{$\begin{array}{l}\text { Grupo genético } \\
\text { Genetic group }\end{array}$} & \multicolumn{4}{|c|}{$\begin{array}{l}\text { Idade (dias) } \\
\text { Age (days) }\end{array}$} \\
\hline & \multicolumn{2}{|c|}{14} & \multicolumn{2}{|c|}{42} \\
\hline & $\mathrm{PC}(\mathrm{g})$ & HCT $(\%)$ & $\mathrm{PC}(\mathrm{g})$ & HCT $(\%)$ \\
\hline & $B W(g)$ & $H C T(\%)$ & $B W(g)$ & HCT (\%) \\
\hline Alto & $243,56 \pm 55,37^{a}$ & $33,75 \pm 3,03^{\mathrm{a}}$ & $1128,17 \pm 149,49^{a}$ & $36,35 \pm 1,93^{\mathrm{a}}$ \\
\hline High & & & & \\
\hline Baixo & $305,59 \pm 54,73^{b}$ & $31,19 \pm 2,98^{b}$ & $1387,06 \pm 193,97^{b}$ & $29,38 \pm 2,29^{b}$ \\
\hline
\end{tabular}

Médias seguidas de letras diferentes na mesma coluna, diferem por Tukey $(P<0,05)$.

Means followed by different letters within a column, differ for the Tukey test $(P<.05)$.

para os dois grupos estudados (Tabela 1) foram inferiores aos padrões de híbridos comerciais AG ROSS ${ }^{\circledR}$ em criações mistas, citados por Abreu et al. (2000), os quais atingiram média de $420 \mathrm{~g}$. Aos 42 dias, as médias obtidas para os dois grupos estudados foram inferiores às determinadas por Silversides et al. (1997), que, trabalhando com nove linhagens comerciais de frangos de corte, obtiveram valores variando de 2108 a $2305 \mathrm{~g}$ de peso vivo.

As médias para o valor hematócrito (Tabela 1) foram significativamente diferentes entre os dois grupos e GAH apresentou as maiores médias $(33,75 \%$ e $36,35 \%$ ) e GBH apresentou médias de $31,19 \%$ e $29,38 \%$, aos 14 e 42 dias, respectivamente.

Shlosberg et al. (1998), trabalhando com três grupos genéticos obtidos de parentais selecionados pelo hematócrito (alto, médio e baixo), verificaram que aves com menor valor hematócrito $(35,50 \%)$ atingiram maior peso corporal $(1802,23 \mathrm{~g})$ e menor mortalidade por ascite (7\%) quando comparado com o grupo alto, em que a média para valor hematócrito foi de 41,55\%; a de peso, de $1623 \mathrm{~g}$; e a mortalidade, de $9 \%$.

Verificou-se que o GAH apresentou maior valor de hematócrito e menor peso corporal nas duas idades estudadas (Tabela 1). Lubritz \& McPherson (1994) e Shlosberg et al. (1998) verificaram existir correlação negativa entre hematócrito e peso corporal. Esta correlação foi explicada por Albers \& Frankenhuis (1990) e Shlosberg et al. (1998) que sugeriram uma possível diminuição no consumo de alimento pelas aves que exibiram maior valor do hematócrito, nas quais, o metabolismo oxidativo torna-se debilitado pelo processo ascítico, quando utilizaram frangos de corte com alta taxa de crescimento

R. Bras. Zootec., v.31, n.3, p.1396-1402, 2002 (suplemento) e criados em temperaturas em torno de $10^{\circ} \mathrm{C}$. Porém, Rosário et al. (2000b) também obtiveram a mesma correlação negativa sem mortalidade por ascite em aves com padrão de crescimento inferior ao de híbridos comerciais, confirmando os relatos da literatura que, para se obter incidência de ascite, deve-se trabalhar com aves de elevada taxa de crescimento. Kubena et al. (1972) verificaram que, em dietas contendo aumento nos níveis dos minerais cobre e ferro, o valor hematócrito foi significativamente superior em frangos criados entre as temperaturas de 21 e $32,2^{\circ} \mathrm{C}$.

As explicações fisiológicas para a diminuição do ganho de peso de aves que apresentam maior valor do hematócrito foram muito bem propostas por Decuypere et al. (2000) os quais relataram que a alta velocidade metabólica dos frangos de corte, selecionados para crescimento, combinado com a seleção para melhoramento da conversão alimentar não suporta o suficiente e regular suprimento de oxigênio para que a capacidade de fosforilação oxidativa seja mantida inalterada. Como a seleção para conversão alimentar continua a ser transferida para as gerações seguintes, tem como uma das conseqüências a diminuição da velocidade metabólica de manutenção. Isto pode ter resultado em uma velocidade de crescimento menor ou em um desbalanceado desenvolvimento dos órgãos que demandam por oxigênio (músculos) e os órgãos que suprem este oxigênio (coração e pulmões), pelo menos durante a rápida fase de crescimento juvenil. O fato dos tecidos cardíacos e pulmonares terem muitas vezes uma velocidade metabólica maior por unidade de peso do que o músculo esquelético, pode reforçar o retardo no desenvolvi- 
mento dos tecidos vitais que suprem oxigênio dentro de uma pressão de seleção combinada com rápida velocidade de crescimento e conversão alimentar. Entretanto, estes tecidos vitais têm de sustentar a alta velocidade metabólica ligada a rápida velocidade de crescimento juvenil garantindo desenvolvimento normal da ave. Quando existe um desbalanço entre a demanda e o suprimento de oxigênio para atender esta velocidade metabólica de crescimento, o quadro ascítico se instala.

É importante ressaltar que durante todo o período experimental não foi possível diagnosticar aves com sintomas externos do quadro ascítico, tais como: acúmulo de fluido ascítico e diminuição no crescimento, não podendo dessa forma afirmar que o GAH é suscetível e o GBH resistente à ascite.

Na Tabela 2 é apresentada a comparação de médias para as características reprodutivas avaliadas nas duas incubações.

Verifica-se pela Tabela 2 que a técnica do microhematócrito não distinguiu os grupos genéticos para as características reprodutivas.

As médias gerais para fertilidade de 93,85 e $94,90 \%$ respectivamente, para GAH e GBH, foram superiores as encontradas por Schmidt et al. (1991a,b) e Ledur et al. (1994), que observaram fertilidade média de $90 \%$ em linhas de macho e de fêmea e de
81,46 e $90,94 \%$ para duas linhas fêmea de matrizes de corte, respectivamente. Porém, essas porcentagens foram inferiores as encontradas por Coelho (1997) que verificou média geral de $95,78 \%$ de fertilidade em linhagens experimentais de matrizes de corte.

As médias gerais obtidas para eclodibilidade de 98,25 e 93,90\%, respectivamente para GAH e GBH, foram superiores as determinadas por Schmidt (1991a,b), Ledur et al. (1994) e Coelho (1997) que encontraram médias de 89 e $92 \%$ de eclodibilidade em linhas de macho e fêmea de matrizes para corte, 82,24 a $86,80 \%$ para linhagens da linha fêmea de matrizes de corte e 76,28 a $86,92 \%$ para linhagens experimentais de matrizes de corte, respectivamente.

Para a característica porcentagem de nascimento, a comparação das médias gerais 89,00 e $82,45 \%$, obtidas respectivamente para GAH e GBH, mostrou ser superior as determinadas por Costa (1980) e Custódio (1981), que obtiveram, respectivamente, médias variando entre 69,40 a $74,10 \%$ e 66,80 a $74,70 \%$ de nascimento, em populações experimentais de matrizes para corte, respectivamente. Porém, as mesmas foram próximas e até superiores as determinadas por Schmidt et al. (1991b) e Coelho (1997), que encontraram médias variando de 83,00 a $84,38 \%$ e 73,30 a $85,08 \%$ de nascimento, respectivamente.

Tabela 2 - Médias e intervalos de confiança das porcentagens de fertilidade, eclodibilidade e nascimento de pintos nos grupos de alto $(\mathrm{GAH})$ e baixo $(\mathrm{GBH})$ hematócrito nas duas incubações/idades

Table 2 - Means and confidence interval of percentages of fertility, hatchability of fertile eggs and overall hatchability in high (HHG) and low hematocrit group (LHG) in two different hatches/ages

\begin{tabular}{|c|c|c|c|c|c|c|c|}
\hline \multirow[t]{2}{*}{$\begin{array}{l}\text { Característica } \\
\text { Characteristic }\end{array}$} & \multirow[t]{2}{*}{$\begin{array}{l}\text { Grupo genético } \\
\text { Genetic group }\end{array}$} & \multicolumn{3}{|c|}{$\begin{array}{l}1^{\mathrm{a}} \text { Incubação/ } 180 \text { dias } \\
1^{s t} \text { Hatch/180 days }\end{array}$} & \multicolumn{3}{|c|}{$\begin{array}{l}2^{\mathrm{a}} \text { Incubação / } 187 \text { dias } \\
2^{\text {nd }} \text { Hatch / } 187 \text { days }\end{array}$} \\
\hline & & $\begin{array}{l}\text { Média }^{1} \\
\text { Mean }^{1}\end{array}$ & & $\begin{array}{l}\mathrm{IC}^{2} \\
C I^{2}\end{array}$ & $\begin{array}{l}\text { Média }^{1} \\
\text { Mean }^{1}\end{array}$ & & \\
\hline Fertilidade (\%) & Alto & 97,2 & 91,2 & 99,9 & 90,5 & 79,4 & 97,7 \\
\hline Fertility (\%) & $\begin{array}{l}\text { High } \\
\text { Baixo }\end{array}$ & 93,7 & 86,2 & 98,3 & 96,1 & 87,5 & 99,9 \\
\hline Low & & & & & & & \\
\hline $\begin{array}{l}\text { Eclodibilidade }(\%) \\
\text { Hatchability of fertile egos } \%)\end{array}$ & $\begin{array}{l}\text { Alto } \\
\text { High }\end{array}$ & 98,2 & 93,4 & 100,0 & 98,3 & 92,3 & 99,9 \\
\hline 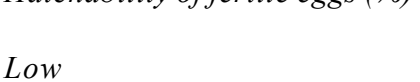 & Baixo & 95,8 & 89,5 & 99,2 & 92,0 & 81,8 & 98,3 \\
\hline $\begin{array}{l}\text { Nascimento (\%) } \\
\text { Overall hatchability (\%) }\end{array}$ & $\begin{array}{l}\text { Alto } \\
\text { High }\end{array}$ & 93,8 & 85,3 & 98,8 & 84,8 & 69,2 & 95,6 \\
\hline 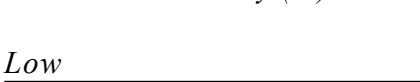 & Baixo & 81,7 & 70,3 & 90,6 & 83,2 & 66,8 & 94,8 \\
\hline
\end{tabular}

${ }^{1}$ Médias não significativas pelo teste do qui-quadrado $(\mathrm{P}<0,05)$.

2 IC: intervalo de $95 \%$ de confiança para a média.

${ }_{1}$ Means do not differ significantly $(P<.05)$.

${ }^{2} \mathrm{Cl}$ : confidence interval $95 \%$ for the mean.

\section{R. Bras. Zootec., v.31, n.3, p.1396-1402, 2002 (suplemento)}


Os valores médios observados para as características reprodutivas foram próximos dos relatados na literatura para ambos os grupos genéticos, o mesmo não ocorrendo para a característica peso corporal, para a qual as aves desses grupos devem ser selecionadas mais intensamente.

Quando a síndrome ascítica se instala, os níveis hormonais de T3 e T4 se alteram, como estudaram Buys et al. (1993), Gonzales et al. (1999) e Decuypere et al. (2000), os quais verificaram que os frangos de corte melhorados para maior taxa de crescimento podem apresentar alteração do metabolismo tiroidiano, em relação as aves não selecionadas. O hipotiroidismo funcional, sugerido por níveis plasmáticos de T3 (triiodotironina) e T4 (tiroxina) alterados, poderia determinar uma redução do consumo de oxigênio tecidual e portanto, predispor as aves a hipoxemia, hipertensão pulmonar, hipertrofia ventricular direita e exsudação de fluido ascítico.

Assim Ringer (1976) relatou que aves com baixo nível de T4 e obesas retardariam o desenvolvimento sexual ou levariam maior tempo para atingir a maturidade sexual, diminuindo, portanto, o desempenho reprodutivo, pois o hipotiroidismo interferiria na liberação em quantidade e equilíbrio de outros hormônios

Pela escassez de trabalhos científicos relacionados ao objetivo proposto, a contribuição deste trabalho para a ciência, além da inovação, seria a difusão da técnica do micro-hematócrito pela avicultura industrial, mais precisamente, para os programas de melhoramento genético de aves.

Este trabalho sugere também outros trabalhos que utilizem de linhagens comerciais de frangos de corte, onde a taxa de crescimento é superior a verificada pelas aves deste trabalho, podendo-se dessa forma, tentar associar a incidência de síndrome ascítica com peso corporal e características reprodutivas, visando a obtenção de materiais genéticos superiores.

\section{Conclusões}

A técnica do micro-hematócrito pode ser recomendada para monitoramento do peso corporal por possibilitar a distinção dos dois grupos genéticos utilizados neste trabalho, onde as aves selecionadas para baixo hematócrito foram mais pesadas que as aves selecionadas para alto hematócrito, porém para as características reprodutivas esta técnica não se mostrou conclusiva.

R. Bras. Zootec., v.31, n.3, p.1396-1402, 2002 (suplemento)

\section{Agradecimento}

Ao professor Dr. Raul Machado Neto, do Departamento de Produção Animal da ESALQ/USP, pelo auxílio nas análises laboratoriais, e ao professor Dr. Sílvio Sandoval Zocchi, do Departamento de Ciências Exatas da ESALQ/USP, pelo auxílio nas análises estatísticas.

\section{Literatura Citada}

ABREU, P.G.; BAÊTA, F.C.; ABREU, V.M.N. et al. Desempenho produtivo e bioeconômico de frangos de corte criados em diferentes sistemas de aquecimento. Revista Brasileira de Zootecnia, v.29, n.1, p.159-167, 2000.

ALBERS, G.; FRANKENHUIS, M. Ascites, a high altitude disease in the lowlands. Poultry Misset, v.6, p.24-25, 1990.

ALBERS, G.A.A. Future trends in poultry breeding. In: EUROPEAN POULTRY CONFERENCE, 10., 1998, Jerusalem, Israel. Proceedings... Jerusalem: WPSA, 1998, p. 16-20.

BURTON, R.R.; SMITH, A.H. Effect of chronic erythrocyte polycythemia and high altitude upon plasma and blood volumes. Proceedings of the Society for Experimental Biology and Medicine, v.140, n.3, p.920-923, 1972.

BUYS, N.; BARTHA, T.; BUYSE, J. et al. Effect of dietary T3 supplementation on ascites incidence in an ascites-sensitive and resistant broiler line: influence on liver deiodination activity and on hormonal profiles in plasma, pericardial and abdominal ascitic fluid. In: SYMPOSIUM ON POULTRY NUTRITION, 9., 1993, Jelenia Góra, Poland. Proceedings... 1993. p.462-467.

COELHO, A.A.D. Análise genética da taxa de crescimento e do desempenho reprodutivo em galinhas de corte. Piracicaba: Luiz de Queiroz, 1997. 74p. Tese (Doutorado em Genética e Melhoramento de Plantas) - Escola Superior de Agricultura Luiz de Queiroz, 1997.

COSTA, M.N. Parâmetros genéticos e fenotípicos para caracteres reprodutivos em galinhas de corte. Piracicaba: Luiz de Queiroz, 1980. 61p. Dissertação (Mestrado em Genética e Melhoramento de Plantas) - Escola Superior de Agricultura Luiz de Queiroz, 1980.

CUSTÓDIO, R.W.S. Análise e interpretação da variabilidade fenotípica para eficiência reprodutiva em galinhas para corte. Piracicaba: Luiz de Queiroz, 1981. 126p. Tese (Livre-Docência) - Escola Superior de Agricultura Luiz de Queiroz, 1981.

DECUYPERE, E.; BUYSE, J.; BUYS, N. Ascites in broiler chickens: exogenous and endogenous structural and functional causal factors. World's Poultry Science Journal, v.56, n.4, p.367-377, 2000

GONZALES, E.; BUYSE, J.; SARTORI, J.R. et al. Metabolic disturbances in male broilers of different strains. 2. Relationship between the thyroid and somatotrophic axes with growth rate and mortality. Poultry Science, v.78, n.2, p.516-521, 1999.

GONZALES, E.; MACARI, M. Enfermidades metabólicas em frangos de corte. In: BERCHIERI Jr., A.; MACARI, M. (Eds.) Doenças das aves. Campinas: Fundação Apinco de Ciência e Tecnologia Avícolas, 2000. p.451-464. 
KUBENA, L.F.; MAY, J.D.; REECE, F.N. et al. Hematocrit and hemaglobin of broilers as influenced by environmental temperature and dietary iron level. Poultry Science, v.51, n.3, p.759-763, 1972.

LEDUR, M.C.; SCHMIDT, G.S.; FIGUEIREDO, E.A.P. et al. Parâmetros genéticos e fenotípicos em linhagens de aves selecionadas para corte. Pesquisa Agropecuária Brasileira, v.29, n.3, p.503-509, 1994.

LUBRITZ, D.L.; McPHERSON, B.N. Effect of genotype and cold stress on incidence of ascites in cockerels. Journal of Applied Poultry Research, v.3, n.2, p.171-178, 1994.

MAXWELL, M.H.; ROBERTSON, G.W.; McCORQUODALE, C.C. Whole blood and plasma viscosity values in normal and ascitic broiler chickens. British Poultry Science, v.33, n.4, p.871-877, 1992.

NATIONAL RESEARCH COUNCIL. Nutrient requirements of poultry. 9.ed. Washington, D.C.: National Academy Press, 1994. 71p.

NIRMALAN, G.P.; ROBINSON, G.A. Hematology of japanese quail treated with exogenous stibestrol dispropionate and testoterone propionate. Poultry Science, v.51, n.3, p.920925, 1972.

RINGER, R.K. Thyroids. In: STURKIE, P.D. (Ed.) Avian physiology. New York: Springer Verlag, 1976. p.348-371.

ROSÁRIO, M.F.; SILVA, M.A.N.; MARTINS, E. et al. Influência do genótipo e do sexo sobre o valor hematócrito em galinhas reprodutoras pesadas. Revista Brasileira de Ciência Avícola, v.2, n.3, p.281-286, 2000a.

ROSÁRIO, M.F.; SILVA, M.A.N.; MARTINS, E. et al. Determinação da idade de seleção através da utilização de regressão linear em frangos de corte. In: SIMPÓSIO INTERNACIONAL DE INICIAÇÃO CIENTÍFICA DA UNIVERSIDADE DE SÃO PAULO, 8., 2000, Piracicaba. Anais... Piracicaba: 2000b. p.116.

SANCHEZ, A.; PLOZEAU, M.; RAULT, P. et al. Croissance musculaire et fonction cardio-respiratoire chez le poulet de chair. INRA Productions Animales, v.13, n.1, p.37-45, 2000.
SAS INSTITUTE SAS OnlineDoc ${ }^{\circledR}$. Version 8, Cary: 1999. SCHMIDT, G.S.; PACKER, I.U.; DUARTE, F.A.M. et al. Formação de populações-bases de aves para corte. III. Avaliação das características reprodutivas, linha macho. Pesquisa Agropecuária Brasileira, v.26, n.6, p.785-790, $1991 \mathrm{a}$.

SCHMIDT, G.S.; PACKER, I.U.; DUARTE, F.A.M. et al. Formação de populações-bases de aves para corte. IV. Avaliação das características produtivas e reprodutivas, linha fêmea. Pesquisa Agropecuária Brasileira, v.26, n.6, p.791796, 1991b.

SHLOSBERG, A.; BELLAICHE, M.; BERMAN, E. et al. Relationship between broiler chicken haematocrit-selected parents and their progeny, with regard to haematocrit, mortality from ascites and body weight. Research in Veterinary Science, v.64, n.2, p.105-109, 1998.

SHLOSBERG, A.; ZADIKOV, I.; BENDHEIM, U. et al. The effects of poor ventilation, low tempertaures, type of feed and sex of bird on the development of ascites in broilers. Physiological factors. Avian Pathology, v.21, n.3, p.369382, 1992.

SILVERSIDES, F.G.; LEFRANÇOIS, M.F.; VILLENEUVE, P. The effect of strain of broiler on physiological parameters associated with the ascites syndrome. Poultry Science, v.76, n.5, p.663-667, 1997.

STURKIE, P.D.; GRIMINGER, P. Body fluids: blood. In: STURKIE, P.D. (Ed.) Avian physiology. New York: Springer Verlag. 1986. p.102-129.

WASHBURN, K.W.; SMYTH Jr., J.R. Hematology of an inherited anemia in the domestic fowl. Poultry Science, v.47, n.1, p.1406-1411, 1968.

Recebido em: 20/02/01

Aceito em: 03/04/02 